\title{
Quantization of surface plasmon polariton by Green's tensor method in amplifying and attenuating media
}

\author{
Z. Allameh \\ Institute of Laser and Plasma, \\ Shahid Beheshti University, Tehran, Iran \\ R. Roknizadeh \\ Department of Physics, Quantum Optics Group \\ University of Isfahan, Isfahan, Iran \\ R. Masoudi \\ Institute of Laser and Plasma, \\ Shahid Beheshti University, Tehran, Iran
}

(Dated: May 30, 2022)

\begin{abstract}
In this paper we will present a quantization method for SPP (Surface Plasmon Polariton) based on Green's tensor method, which is applied usually for quantization of EM field in various dielectric media. This method will be applied for a semi-infinite structure, which contains metal and dielectric regions with one interface. Moreover, by introducing the quantized SPP, we will investigate the SPP propagation in the attenuating and amplifying systems. We will also consider two modes of $\mathrm{SPP}$, i.e., coherent and squeezed states, and finally compare the propagation of these modes in the amplifying media.
\end{abstract}

PACS: $42.50 .-\mathrm{p}$ 


\section{INTRODUCTION}

A SPP is a an electromagnetic 2-dimensional excitation existing on the metal-dielectric interface, which decays exponentially with diffusing depth in the metal. The term "surface plasmon" refers to the collective electrons oscillation on the metal surface, and "polariton", which combine exciton and photon properties as a quasi-particle, indicates that the SPP involves coupling of electromagnetic waves and the dielectric excitations. Due to the unique properties of the SPP, such as capacity of confining the electromagnetic field [1, 2], it has become one of the interesting field of applied research [3].

By discovering the quantum behavior of SPP in theoretical and experimental investigations [4-7], the possibility of using them in quantum technology, such as Quantum Information Processing (QIP) [8-10] and generating and controlling plasmonic excitation at the quantum level, has been provided $[11,12]$.

In order to describe the plasmonic quantum mechanically, a quantization method of SPP is introduced in [13]. This formalism is based on Hopfield approach and therefore do not consider the dissipation within the medium. It is well known that the real part of permittivity is related, by the Kramers-Kronig relation, to the stored energy or dispersion within medium, and imaginary part yields the dissipation (or loss) of the energy within it. For lossy and dispersive dielectric, Huttner and Barnett have proposed a microscopic quantization method [14] . In their method the absorptive medium is assumed to be connected to a reservoir and represented by a collection of interacting matter fields.

As another most interesting scheme for quantization, the Greens function method have been proposed and used by many authors [15-18]. The Greens function is constructed for classical Maxwell equations and contains, as a whole, the system and the source of the noise associated with the absorption of radiation in it [19-22].

In this contribution we will try to apply this method for quantization of SPP field. Moreover, we apply this approach to propagation of the SPP in the amplifying media. Nowadays, because of the significant applications of this field, many efforts have been devoted to the SPP amplification [23-28]. The quantum approach not only enables us to take into account the SPP amplification, but also the behavior of SPP's different states can be distinguished $[29]$.

The paper is organized as follows. In section 2 we review the quantization of EM-field in 
dielectric media. Regarding to the SPP propagation along the interface of metal-dielectric, we study a single flat interface system in section 3. In section 4 we apply this approach for investigating the SPP propagation in attenuating and amplifying system for coherent and squeezed states of SPP. A conclusions is given in sec 5 .

\section{BASIC CONCEPTS}

In order to obtain a suitable form of the Maxwell equations for quantization procedure, we review the basic concepts in classical and quantum electrodynamics [15-18].

At first, it is useful to express electromagnetic fields in frequency domain, and separate them in negative and positive frequency components. For instance the electric field is written in the form,

$$
E(r, \omega)=E^{+}(r, \omega)+E^{-}(r, \omega)
$$

here

$$
E^{+}(r, t)=\frac{1}{\sqrt{2 \pi}} \int_{0}^{+\infty} \mathrm{d} \omega E^{+}(r, \omega) \exp (-i \omega t)
$$

and the other vector fields are expressed analogously. Then, in a medium without free

charges and currents, the classical maxwell equations for positive part in the frequency domain are given by,

$$
\begin{aligned}
\nabla \cdot D^{+}(r, \omega) & =0, \\
\nabla \cdot B^{+}(r, \omega) & =0, \\
\nabla \times E^{+}(r, \omega) & =i \omega B^{+}(r, \omega), \\
\nabla \times H^{+}(r, \omega) & =-i \omega D^{+}(r, \omega),
\end{aligned}
$$

where $D^{+}(r, \omega)$ and $B^{+}(r, \omega)$ satisfy the constitutive relations:

$$
\begin{aligned}
& D^{+}(r, \omega)=\epsilon_{0} \epsilon(r, \omega) E^{+}(r, \omega), \\
& B^{+}(r, \omega)=\mu_{0} H^{+}(r, \omega) .
\end{aligned}
$$

where $\epsilon(r, \omega)$ is complex dielectric function, its real and imaginary parts are related by Kramers- Kronig relation. Also, $\epsilon(r, \omega)$ is related to complex refractive index, $n(r, \omega)$,

$$
\epsilon(r, \omega)=[n(r, \omega)]^{2}
$$


where

$$
n(r, \omega)=\eta(r, \omega)+i \kappa(r, \omega) .
$$

In frequency domain, we have the following relations,

$$
\epsilon(r,-\omega)=\epsilon(r, \omega)^{*}, \quad n(r,-\omega)=n(r, \omega)^{*} .
$$

As a general property for $\epsilon(r, \omega)$ it is, in the whole upper half of the complex frequency plane, an analytic function without zeros. So that, with respect to the causality, $\epsilon(r, \omega)$ has asymptotic behavior for high frequency [30, 31],

$$
\epsilon(r, \omega) \rightarrow 1, \quad \text { for } \quad \omega \rightarrow \infty
$$

In quantizing the EM-vector fields and constitutive relations (4) and (5), we have to take into account the fluctuation- dissipation theorem. According to this theorem, an unavoidable consequence of the radiation absorption (or dissipation) in reservoir, is injection the additional noise (like thermal radiation) from environment to the fields. The noise term can be expressed by introducing a polarization operator in Eq. (4),

$$
\hat{D}^{+}(r, \omega)=\epsilon_{0} \epsilon(r, \omega) \hat{E}^{+}(r, \omega)+\hat{P}_{N}^{+}(r, \omega) .
$$

So the quantized Maxwell equations are obtained as:

$$
\begin{aligned}
& \nabla \cdot\left[\epsilon_{0} \epsilon(r, \omega) \hat{E}^{+}(r, \omega)\right]=\hat{\rho}_{N}^{+}(r, \omega), \\
& \nabla \cdot \hat{B}^{+}(r, \omega)=0, \\
& \nabla \times \hat{E}^{+}(r, \omega)=i \omega \hat{B}^{+}(r, \omega), \\
& \nabla \times \hat{B}^{+}(r, \omega)=-i \omega \mu_{0} \epsilon_{0} \epsilon(r, \omega) \hat{E}^{+}(r, \omega)+\mu_{0} \hat{j}_{N}^{+}(r, \omega),
\end{aligned}
$$

here $\hat{\rho}_{N}^{+}(r, \omega)$ and $\hat{j}_{N}^{+}(r, \omega)$ are noise charge density and noise current density respectively which are related to $\hat{P}_{N}^{+}(r, \omega)$ by,

$$
\begin{aligned}
& \hat{\rho}_{N}^{+}(r, \omega)=-\nabla \cdot \hat{P}_{N}^{+}(r, \omega), \\
& \hat{j}_{N}^{+}(r, \omega)=-i \omega \hat{P}_{N}^{+}(r, \omega) .
\end{aligned}
$$

It is suitable to obtain a partial differential equation for $\hat{A}(r, \omega)$. By expressing the field operators in terms of vector potential operator $\hat{A}(r, \omega)$ :

$$
\begin{aligned}
& \hat{E}^{+}(r, \omega)=i \omega \hat{A}^{+}(r, \omega), \\
& \hat{B}^{+}(r, \omega)=\nabla \times \hat{A}^{+}(r, \omega) .
\end{aligned}
$$


and applying Eqs. (13) and (14), one obtains:

$$
-\nabla \times \nabla \times \hat{A}^{+}(r, \omega)+\frac{\omega^{2}}{c^{2}} \epsilon(r, \omega) \hat{A}^{+}(r, \omega)=-\mu_{0} \hat{j}_{N}^{+}(r, \omega) .
$$

A standard method for solving this equation is using the Green's tensor,

$$
\hat{A}^{+}(r, \omega)=-\mu_{0} \int_{-\infty}^{+\infty} \mathrm{d} r^{\prime} G\left(r, r^{\prime}, \omega\right) \cdot \hat{j}_{N}^{+}\left(r^{\prime}, \omega\right),
$$

and therefore it has to solve the following equation,

$$
-\nabla \times \nabla \times G\left(r, r^{\prime}, \omega\right)+\frac{\omega^{2}}{c^{2}} \epsilon(r, \omega) G\left(r, r^{\prime}, \omega\right)=I \delta\left(r-r^{\prime}\right) .
$$

where $I$ is the unit tensor. The Green's tensor has some general properties, which are given in appendix A. In the field quantization one requires the vector fields satisfying the canonical commutation relations at any point of space,

$$
\left[\hat{A}_{i}(r, t),-\epsilon_{0} \hat{E}_{j}\left(r^{\prime}, t\right)\right]=i \hbar \delta\left(r-r^{\prime}\right) \delta_{i j}
$$

The main reason for accuracy of this relation in absorbing media is the noise sources. The commutation relation for the noise current operators is given by:

$$
\begin{aligned}
& {\left[\hat{j}_{N}^{(+)}(r, \omega), \hat{j}_{N}^{(-)}\left(r^{\prime}, \omega^{\prime}\right)\right]=|\alpha(\omega)| \delta\left(r-r^{\prime}\right) \delta\left(\omega-\omega^{\prime}\right),} \\
& {\left[\hat{j}_{N}^{(+)}(r, \omega), \hat{j}_{N}^{(+)}\left(r^{\prime}, \omega^{\prime}\right)\right]=\left[\hat{j}_{N}^{(-)}(r, \omega), \hat{j}_{N}^{(-)}\left(r^{\prime}, \omega^{\prime}\right)\right]=0 .}
\end{aligned}
$$

where $\alpha(\omega)$ is determined such that Eqs.(20) and (21) be consistent with each other. On the other hand the noise current is related to Langevin operator,

$$
\hat{j}_{N}^{(+)}(r, \omega)=\sqrt{\alpha(\omega)} \hat{f}(r, \omega)
$$

Also one can obtain the propagators of noise current [15-17],

$$
\begin{aligned}
& \left\langle 0\left|\hat{j}_{N}^{(+)}(r, \omega) \hat{j}_{N}^{(+)}\left(r^{\prime}, \omega^{\prime}\right)\right| 0\right\rangle=\left\langle 0\left|\hat{j}_{N}^{(-)}(r, \omega) \hat{j}_{N}^{(-)}\left(r^{\prime}, \omega^{\prime}\right)\right| 0\right\rangle=0 \\
& \left\langle 0\left|\hat{j}_{N}^{(-)}(r, \omega) \hat{j}_{N}^{(+)}\left(r^{\prime}, \omega^{\prime}\right)\right| 0\right\rangle=0 \\
& \left\langle 0\left|\hat{j}_{N}^{(+)}(r, \omega) \hat{j}_{N}^{(-)}\left(r^{\prime}, \omega^{\prime}\right)\right| 0\right\rangle=|\alpha(\omega)| \delta\left(r-r^{\prime}\right) \delta\left(\omega-\omega^{\prime}\right)
\end{aligned}
$$

\section{QUANTIZATION OF THE SPP FIELD ON A METAL- DIELECTRIC IN- TERFACE}

In this section we consider the quantized field of SPP, which propagates along the metaldielectric interface lying in $x y$-plane, as shown schematically in Fig.1. For simplicity we 


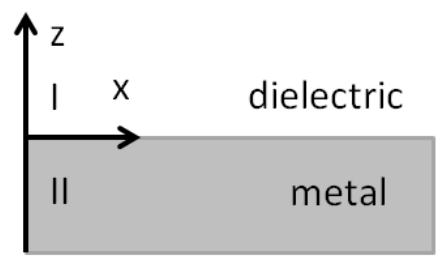

FIG. 1. Semi-infinite media, SPP propagates along the interface of metal- dielectric.

assume that the both media are homogeneous, so that the related dielectric constants are independent of space points. The dielectric constant corresponding to the whole space is written as,

$$
\epsilon(r, \omega)=\epsilon_{m}(\omega) \Theta(-z)+\epsilon_{d}(\omega) \Theta(z)
$$

here $\epsilon_{m}(\omega)$ and $\epsilon_{d}(\omega)$ are dielectric functions of metal and dielectric media, respectively and $\Theta$ is the step function. In order to use the Eqs.(17) and (18) as starting points for quantization process, we must derive the Green's tensor.

\section{A. Construction of the Green's tensor}

For construction of the Green's tensor we use the method of eigenmode expansion [3234]. In this approach, we consider the generalized form of Eq.(17), which contains a set of eigenmodes and eigenvalues $\left(A_{n}, \lambda_{n}\right)$,

$$
-\nabla \times \nabla \times A_{n}(r)+\frac{\omega^{2}}{c^{2}} \epsilon(r, \omega) A_{n}(r)=\epsilon(r, \omega) \lambda_{n} A_{n}(r) .
$$

These eigenmodes satisfy the orthogonality condition:

$$
\int_{-\infty}^{+\infty} \epsilon(r, \omega) A_{n}(r) \cdot\left[A_{m}(r)\right]^{*} \mathrm{~d}^{3} r=\mathrm{N}_{n} \delta_{n m}
$$

By obtaining the explicit form of eigenmodes and calculating the eigenvalues and normalization factor from Eqs. (24) and (25), the Green's tensor is given by,

$$
G\left(r, r^{\prime}\right)=\sum_{n} \frac{A_{n}(r)\left[A_{n}\left(r^{\prime}\right)\right]^{*}}{\mathrm{~N}_{n} \lambda_{n}}
$$


By this method the Green's tensor, for geometry shown in Fig 1 can be derived. The SPP's eigenmodes can be written,

$$
\begin{aligned}
& A_{k_{x}}(x, z)=\left(i \hat{x}+\frac{k_{x}}{\nu_{i}} \hat{z}\right) e^{\left(\nu_{i} z\right)} e^{i k_{x} x}, \quad z<0 \\
& A_{k_{x}}(x, z)=\left(i \hat{x}-\frac{k_{x}}{\nu_{0}} \hat{z}\right) e^{\left(-\nu_{0} z\right)} e^{i k_{x} x}, \quad z>0
\end{aligned}
$$

here $\nu_{i}$ and $\nu_{0}$ are out of plane decay constant and are given by:

$$
\begin{aligned}
& \nu_{i}=\sqrt{\frac{-\epsilon_{m}}{\epsilon_{d}}}\left|k_{x}\right|, \\
& \nu_{0}=\sqrt{\frac{-\epsilon_{d}}{\epsilon_{m}}}\left|k_{x}\right| .
\end{aligned}
$$

$k_{x}$ is the in plane propagation wave vector. The details of this calculation have been given in appendix A. By inserting Eq.(27) in to Eqs. (24) and (25), $\lambda_{n}$ and $N_{n}$ are obtained:

$$
\begin{aligned}
\lambda_{n} & =k_{0}^{2}-k_{x}^{2} \frac{\epsilon_{d}+\epsilon_{m}}{\epsilon_{m} \epsilon_{d}} \\
N_{n} & =\frac{\pi}{\left|k_{x}\right|} \sqrt{\frac{-\epsilon_{m}}{\epsilon_{d}}} \frac{\left(\epsilon_{d}^{2}-\epsilon_{m}^{2}\right)}{\epsilon_{m}} .
\end{aligned}
$$

According to Eqs. (26) and (27), for $z, z^{\prime}<0$ and $z>0, z^{\prime}<0$, the Green's tensor can be written as:

$$
\begin{aligned}
& G\left(r, r^{\prime}, \omega_{0}\right)=-C \int_{-\infty}^{\infty} \mathrm{d} k_{x}\left\{\frac{\left|k_{x}\right|}{\left(k_{s p p}^{2}-k_{x}^{2}-i 0^{+}\right)}\right. \\
& {\left[\left(i \hat{x}+\frac{k_{x}}{\nu_{i}} \hat{z}\right) e^{\left(\nu_{i} z\right)} \Theta(-z)+\left(i \hat{x}-\frac{k_{x}}{\nu_{0}} \hat{z}\right) e^{\left(-\nu_{0} z\right)} \Theta(z)\right] \times} \\
& {\left[\left(i \hat{x}+\frac{k_{x}}{\nu_{i}} \hat{z}\right) e^{\left(\nu_{i} z^{\prime}\right)} \Theta\left(-z^{\prime}\right)+\left(i \hat{x}-\frac{k_{x}}{\nu_{0}} \hat{z}\right) e^{\left(-\nu_{0} z^{\prime}\right)} \Theta\left(z^{\prime}\right)\right]} \\
& \left.e^{i k_{x}\left(x-x^{\prime}\right)}\right\},
\end{aligned}
$$

here $\Theta$ is the step function and $i 0^{+}$in the denominator is necessary for obtaining the retarded Green's tensor and:

$$
\begin{aligned}
C & =\frac{\epsilon_{m} \epsilon_{d}}{\left(\epsilon_{d}+\epsilon_{m}\right) \pi \sqrt{\frac{-\epsilon_{m}}{\epsilon_{d}}} \frac{\left(\epsilon_{d}^{2}-\epsilon_{m}^{2}\right)}{\epsilon_{m}}}, \\
k_{s p p}^{2} & =k_{0}^{2} \frac{\epsilon_{m} \epsilon_{d}}{\epsilon_{d}+\epsilon_{m}}=\left(k^{\prime}+k^{\prime \prime}\right)^{2} .
\end{aligned}
$$


By using that $\lim _{\epsilon \rightarrow 0} \frac{1}{y-i \epsilon}=P V \frac{1}{y}+i \pi \delta(y)$ and the residu theorem one can evaluate the integral in Eq.(30). This result in the far- field approximation yields:

$$
\begin{aligned}
& G\left(r, r^{\prime}, \omega_{0}\right)=-i C^{\prime} \times \\
& {\left[\left(i \hat{x}+\frac{k_{s p p}}{\nu_{i}} \hat{z}\right) e^{\left(\nu_{i} z\right)} \Theta(-z)+\left(i \hat{x}-\frac{k_{s p p}}{\nu_{0}} \hat{z}\right) e^{\left(-\nu_{0} z\right)} \Theta(z)\right] \times} \\
& {\left[\left(i \hat{x}+\frac{k_{s p p}}{\nu_{i}} \hat{z}\right) e^{\left(\nu_{i} z^{\prime}\right)} \Theta\left(-z^{\prime}\right)+\left(i \hat{x}-\frac{k_{s p p}}{\nu_{0}} \hat{z}\right) e^{\left(-\nu_{0} z^{\prime}\right)} \Theta\left(z^{\prime}\right)\right]} \\
& e^{i k_{s p p}\left|x-x^{\prime}\right|},
\end{aligned}
$$

where $C^{\prime}=\pi C$.

\section{B. Field quantization and Canonical commutation relations}

By substitution the Green's tensor Eq. (33) into the Eq. (18), the vector potential operator is now obtained,

$$
\begin{aligned}
& \hat{A}^{+}(r, \omega)=i \mu_{0} C^{\prime} \times \\
& {\left[\left(i \hat{x}+\frac{k_{s p p}}{\nu_{i}} \hat{z}\right) e^{\left(\nu_{i} z\right)} \Theta(-z)+\left(i \hat{x}-\frac{k_{s p p}}{\nu_{0}} \hat{z}\right) e^{\left(-\nu_{0} z\right)} \Theta(z)\right] \times} \\
& \int_{-\infty}^{\infty} \int_{-\infty}^{\infty}\left[\mathrm{d} z^{\prime} \mathrm{d} x^{\prime} e^{i k_{s p p} \mid x-x^{\prime}} \mid \hat{j}_{N}^{+}\left(x^{\prime}, z^{\prime}, \omega\right) .\right. \\
& {\left[\left(i \hat{x}+\frac{k_{s p p}}{\nu_{i}} \hat{z}\right) e^{\left(\nu_{i} z^{\prime}\right)} \Theta\left(-z^{\prime}\right)+\left(i \hat{x}-\frac{k_{s p p}}{\nu_{0}} \hat{z}\right) e^{\left(-\nu_{0} z^{\prime}\right)} \Theta\left(z^{\prime}\right)\right] .}
\end{aligned}
$$

The noise current contains two components, which are related to two regions (metal and dielectric),

$$
\begin{aligned}
\hat{j}_{N}^{(+)}(x, z, \omega) & =\hat{j}_{N}^{(+) m}(x, z, \omega) \Theta(-z)+\hat{j}_{N}^{(+) d}(x, z, \omega) \Theta(z) \\
& =\left(\sqrt{\alpha^{m}(\omega)} \Theta(-z)+\sqrt{\alpha^{d}(\omega)} \Theta(z)\right) \hat{f}(x, z, \omega) .
\end{aligned}
$$

It is convenient to separate the vector potential operator into two components, rightwards and leftwards, based on the propagation direction,

$$
\begin{aligned}
& \hat{A}^{+}(r, \omega)=i\left(\frac{\beta(\omega)}{2 k^{\prime \prime}}\right)^{\frac{1}{2}} \mu_{0} C^{\prime} \times \\
& \left\{\left(i \hat{x}+\frac{k_{s p p}}{\nu_{i}} \hat{z}\right) e^{\left(\nu_{i} z\right)} \Theta(-z)+\left(i \hat{x}-\frac{k_{s p p}}{\nu_{0}} \hat{z}\right) e^{\left(-\nu_{0} z\right)} \Theta(z)\right\} \times \\
& {\left[\hat{a}_{R}(x, \omega)+\hat{a}_{L}(x, \omega)\right],}
\end{aligned}
$$


where $\hat{a}_{R}\left(\hat{a}_{L}\right)$ is annihilation operator for rightwards (leftwards) SPP modes and have the explicit form,

$$
\begin{aligned}
& \hat{a}_{R}(x, \omega)=\left(\frac{2 k^{\prime \prime}}{\beta(\omega)}\right)^{\frac{1}{2}} \int_{-\infty}^{\infty} \int_{-\infty}^{x} \mathrm{~d} x^{\prime} \mathrm{d} z^{\prime} e^{i k_{s p p}\left(x-x^{\prime}\right)} \times \\
& {\left[\left(i \hat{x}+\frac{k_{s p p}}{\nu_{i}} \hat{z}\right) e^{\left(\nu_{i} z^{\prime}\right)} \cdot \hat{j}_{N}^{(+) m}(x, z, \omega) \Theta(-z) \Theta\left(-z^{\prime}\right)+\right.} \\
& \left.\left(i \hat{x}-\frac{k_{s p p}}{\nu_{0}} \hat{z}\right) e^{\left(-\nu_{0} z^{\prime}\right)} \cdot \hat{j}_{N}^{(+) d}(x, z, \omega) \Theta(z) \Theta\left(z^{\prime}\right)\right], \\
& \hat{a}_{L}(x, \omega)=\left(\frac{2 k^{\prime \prime}}{\beta(\omega)}\right)^{\frac{1}{2}} \int_{-\infty}^{\infty} \int_{x}^{\infty} \mathrm{d} x^{\prime} \mathrm{d} z^{\prime} e^{-i k_{s p p}\left(x-x^{\prime}\right)} \times \\
& {\left[\left(i \hat{x}+\frac{k_{s p p}}{\nu_{i}} \hat{z}\right) e^{\left(\nu_{i} z^{\prime}\right)} \cdot \hat{j}_{N}^{(+) m}(x, z, \omega) \Theta(-z) \Theta\left(-z^{\prime}\right)+\right.} \\
& \left.\left(i \hat{x}-\frac{k_{s p p}}{\nu_{0}} \hat{z}\right) e^{\left(-\nu_{0} z^{\prime}\right)} \cdot \hat{j}_{N}^{(+) d}(x, z, \omega) \Theta(z) \Theta\left(z^{\prime}\right)\right],
\end{aligned}
$$

where

$$
\beta(\omega)=\left(1+\frac{\left|k_{s p p}\right|^{2}}{\left|\nu_{i}\right|^{2}}\right) \frac{\left|\alpha^{m}(\omega)\right|}{\nu_{i}+\nu_{i}^{*}}+\left(1+\frac{\left|k_{\text {spp }}\right|^{2}}{\left|\nu_{0}\right|^{2}}\right) \frac{\left|\alpha^{d}(\omega)\right|}{\nu_{0}+\nu_{0}^{*}}
$$

By this definition, one can obtain the bosonic commutation relation at the same positions,

$$
\begin{aligned}
& {\left[\hat{a}_{R}(x, \omega), \hat{a}_{R}^{\dagger}\left(x, \omega^{\prime}\right)\right]=\left[\hat{a}_{L}(x, \omega), \hat{a}_{L}^{\dagger}\left(x, \omega^{\prime}\right)\right]=\delta\left(\omega-\omega^{\prime}\right),} \\
& {\left[\hat{a}_{R}(x, \omega), \hat{a}_{L}^{\dagger}\left(x, \omega^{\prime}\right)\right]=\left[\hat{a}_{L}(x, \omega), \hat{a}_{R}^{\dagger}\left(x, \omega^{\prime}\right)\right]=0 .}
\end{aligned}
$$

The representation of the vector potential in quantized scheme Eq.(36) is acceptable when the canonical commutation relation (20) is satisfied simultaneously. In order to show the accuracy of the relation (20), it is advantageous to derive the commutation relations for the creation and annihilation operators at any two points of space along the $x$ direction,

$$
\begin{aligned}
& {\left[\hat{a}_{R}(x, \omega), \hat{a}_{R}^{\dagger}\left(x^{\prime}, \omega^{\prime}\right)\right]=\left[\hat{a}_{L}\left(x^{\prime}, \omega^{\prime}\right), \hat{a}_{L}^{\dagger}(x, \omega)\right]=} \\
& \delta\left(\omega-\omega^{\prime}\right) \exp \left(i k^{\prime}\left(x-x^{\prime}\right)\right) \exp \left(-k^{\prime \prime}\left|x-x^{\prime}\right|\right), \\
& {\left[\hat{a}_{R}(x, \omega), \hat{a}_{L}^{\dagger}\left(x^{\prime}, \omega^{\prime}\right)\right]=\left[\hat{a}_{L}\left(x^{\prime}, \omega^{\prime}\right), \hat{a}_{R}^{\dagger}(x, \omega)\right]=} \\
& \delta\left(\omega-\omega^{\prime}\right) \Theta\left(x-x^{\prime}\right) \frac{2 k^{\prime \prime}}{k^{\prime}} \exp \left(-k^{\prime \prime}\left(x-x^{\prime}\right)\right) \sin k^{\prime}\left(x-x^{\prime}\right) .
\end{aligned}
$$

The relations (39) and (40) are the same such as evaluated in Ref. [15] for field operator in dielectric medium. Matloob et al. in [15], have interpreted these relations, and also mentioned that the presence of the loss leads to coupling the rightwards and leftwards operators. By applying these relations, the canonical commutation relation is written in the 
following form.

$$
\begin{aligned}
& {\left[\hat{A}(r, t),-\epsilon_{0} \hat{E}\left(r^{\prime}, t\right)\right]=\int_{0}^{\infty} \mathrm{d} \omega\left|C^{\prime}\right|^{2} \mu_{0}^{2} \frac{i \omega \epsilon_{0} \beta(\omega)}{2 k^{\prime \prime} \pi} \times} \\
& \frac{\left|k_{s p p}\right|^{2}}{k^{\prime}}\left\{\frac{e^{i k_{s p p}\left|x-x^{\prime}\right|}}{k_{s p p}}+\frac{e^{-i k_{s p p}^{*}\left|x-x^{\prime}\right|}}{k_{s p p}^{*}}\right\} \times \\
& {\left[\left(i \hat{x}+\frac{k_{s p p}}{\nu_{i}} \hat{z}\right) e^{\left(\nu_{i} z\right)} \Theta(-z)+\left(i \hat{x}-\frac{k_{s p p}}{\nu_{0}} \hat{z}\right) e^{\left(-\nu_{0} z\right)} \Theta(z)\right] \times} \\
& {\left[\left(-i \hat{x}+\frac{k_{s p p}^{*}}{\nu_{i}^{*}} \hat{z}\right) e^{\nu_{i}^{*} z^{\prime}} \Theta\left(-z^{\prime}\right)+\left(-i \hat{x}-\frac{k_{s p p}^{*}}{\nu_{0}^{*}} \hat{z}\right) e^{-\nu_{0}^{*} z^{\prime}} \Theta\left(z^{\prime}\right)\right] .}
\end{aligned}
$$

By some calculations, done in appendix B, the convenient form of the above equation can be achieved,

$$
\left[\hat{A}(r, t),-\epsilon_{0} \hat{E}\left(r^{\prime}, t\right)\right]=\int_{0}^{\infty} \mathrm{d} \omega \frac{i \beta(\omega)}{\pi \epsilon_{0} c^{2} \omega \gamma(\omega)} \operatorname{Im} G\left(r, r^{\prime}, \omega\right)
$$

where $c$ is the light velocity and $\gamma(\omega)$ has been introduced in appendix B. By choosing

$$
\beta(\omega)=2 \hbar \epsilon_{0} \omega^{2} \gamma(\omega),
$$

we have,

$$
\left[\hat{A}(r, t),-\epsilon_{0} \hat{E}\left(r^{\prime}, t\right)\right]=\frac{2 i \hbar}{\pi} \int_{0}^{\infty} \mathrm{d} \omega \frac{\omega}{c^{2}} \operatorname{Im} G\left(r, r^{\prime}, \omega\right)
$$

By considering Eq.(62) and Eq.(63) in appendix A, and using the polar coordinate $\omega=$ $|\omega| e^{i \varphi}$, the right hand side of above equation is written as follows,

$$
\begin{aligned}
\int_{0}^{\infty} \mathrm{d} \omega \frac{\omega}{c^{2}} & \operatorname{Im} G\left(r, r^{\prime}, \omega\right)=\frac{1}{2 i} \int_{-\infty}^{\infty} \mathrm{d} \omega \frac{\omega}{c^{2}} G\left(r, r^{\prime}, \omega\right) \\
& =-\frac{1}{2} \lim _{|\omega| \rightarrow \infty} \int_{0}^{\pi} \mathrm{d} \varphi \frac{\omega^{2}}{c^{2}} G\left(r, r^{\prime}, \omega\right) \\
& =\frac{\pi}{2} \delta\left(r-r^{\prime}\right) .
\end{aligned}
$$

By substituting (45) in (44) the explicit form of canonical commutation relation which is identical with Eq.(20), would be obtained,

$$
\left[\hat{A}(r, t),-\epsilon_{0} \hat{E}\left(r^{\prime}, t\right)\right]=i \hbar \delta\left(r-r^{\prime}\right)
$$




\section{The fluctuation relations}

According to the relation (16) and the vector potential operator in (36), the electric field can be written,

$$
\begin{aligned}
& \hat{E}^{+}(r, \omega)=i \omega \hat{A}^{+}(r, \omega)=-\omega\left(\frac{\beta(\omega)}{2 k^{\prime \prime}}\right)^{\frac{1}{2}} \mu_{0} C^{\prime} \times \\
& \left\{\left(i \hat{x}+\frac{k_{s p p}}{\nu_{i}} \hat{z}\right) e^{\left(\nu_{i} z\right)} \Theta(-z)+\left(i \hat{x}-\frac{k_{s p p}}{\nu_{0}} \hat{z}\right) e^{\left(-\nu_{0} z\right)} \Theta(z)\right\} \times \\
& {\left[\hat{a}_{R}(x, \omega)+\hat{a}_{L}(x, \omega)\right] .}
\end{aligned}
$$

Regarding to the relation (22) and appendix B, the electric field fluctuation can be achieved,

$$
\left\langle 0\left|E(r, \omega) E\left(r^{\prime}, \omega^{\prime}\right)\right| 0\right\rangle=2 \hbar \omega^{2} \mu_{0} \operatorname{Im} G\left(r, r^{\prime}, \omega\right) \delta\left(\omega-\omega^{\prime}\right) .
$$

On the other hand from (43) one can deduce the form of the $\alpha^{m}(\omega)$ and $\alpha^{d}(\omega)$ in terms of the media parameters,

$$
\begin{aligned}
& \left|\alpha^{m}(\omega)\right|=2 \hbar \omega^{2} \epsilon_{0} \operatorname{Im} \epsilon_{m}(\omega), \\
& \left|\alpha^{d}(\omega)\right|=2 \hbar \omega^{2} \epsilon_{0} \operatorname{Im} \epsilon_{d}(\omega) .
\end{aligned}
$$

By applying above relations, the noise current fluctuation is given by,

$$
\begin{aligned}
& \left\langle 0\left|j(r, \omega) j\left(r^{\prime}, \omega^{\prime}\right)\right| 0\right\rangle=\delta\left(r-r^{\prime}\right) \delta\left(\omega-\omega^{\prime}\right) \\
& 2 \hbar \omega^{2} \epsilon_{0}\left(\operatorname{Im} \epsilon_{m}(\omega) \Theta(-z)+\operatorname{Im} \epsilon_{d}(\omega) \Theta(z)\right) .
\end{aligned}
$$

These results agree with those calculated by the fluctuation-dissipation theorem.

\section{THE MAGNETIC FIELD VARIATION IN AMPLIFYING AND ATTENUAT- ING MEDIA FOR COHERENT AND SQUEEZED SPP MODES}

\section{A. Magnetic field}

In order to express the magnetic field by using (16) and (36), the following relations are necessary,

$$
\begin{aligned}
& \frac{\partial \hat{a}_{R}(x, \omega)}{\partial x}=i k_{s p p} \hat{a}_{R}(x, \omega)+\hat{F}(x, \omega), \\
& \frac{\partial \hat{a}_{L}(x, \omega)}{\partial x}=-i k_{s p p} \hat{a}_{L}(x, \omega)-\hat{F}(x, \omega),
\end{aligned}
$$


where

$$
\begin{aligned}
\hat{F}(x, \omega) & =\left(\frac{2 k^{\prime \prime}}{\beta(\omega)}\right)^{\frac{1}{2}} \int_{-\infty}^{\infty} \mathrm{d} z^{\prime}\left[\left(i \hat{x}+\frac{k_{s p p}}{\nu_{i}} \hat{z}\right) e^{\left(\nu_{i} z^{\prime}\right)} \cdot \hat{j}_{N}^{m+}(x, z, \omega) \Theta(-z) \Theta\left(-z^{\prime}\right)\right. \\
& \left.+\left(i \hat{x}-\frac{k_{s p p}}{\nu_{0}} \hat{z}\right) e^{\left(-\nu_{0} z^{\prime}\right)} \cdot \hat{j}_{N}^{d+}(x, z, \omega) \Theta(z) \Theta\left(z^{\prime}\right)\right]
\end{aligned}
$$

The equations (51) are the well-known quantum Langevin equations. The $\hat{F}(x, \omega)$ is the operator of Langevin noise source and satisfies the following commutation relation,

$$
\left[\hat{F}(x, \omega), \hat{F}\left(x^{\prime}, \omega^{\prime}\right)\right]=2 k^{\prime \prime} \delta\left(x-x^{\prime}\right) \delta\left(\omega-\omega^{\prime}\right) .
$$

Therefore, the magnetic field operators of the propagating rightwards SPP modes are,

$$
\begin{aligned}
& \hat{H}^{+}(r, \omega)=-\left(\frac{\beta(\omega)}{2 k}\right)^{\frac{1}{2}} C^{\prime} \hat{a}_{R}(x, \omega) \times \\
& \left\{\left(\nu_{i}+\frac{k_{s p p}^{2}}{\nu_{i}}\right) e^{\left(\nu_{i} z\right)} \Theta(-z)-\left(\nu_{0}+\frac{k_{s p p}^{2}}{\nu_{0}}\right) e^{\left(-\nu_{0} z\right)} \Theta(z)\right\} .
\end{aligned}
$$

On the other hand, the explicit solution for (51) can be derived,

$$
\hat{a}_{R}(x, \omega)=e^{i k_{s p p}\left(x-x^{\prime}\right)} \hat{a}_{R}\left(x^{\prime}, \omega\right)+\int_{x^{\prime}}^{x} \hat{F}(y, \omega) e^{i k_{s p p}(x-y)} \mathrm{d} y,
$$

where $x \geq x^{\prime}$. This equation connects two operators in different points of space with each other. By applying the general property of the noise operator $\langle f(x, \omega)\rangle=0$, the average of $\hat{a}_{R}(x, \omega)$, at $x^{\prime}=0$, the starting point of rightwards propagation, is given by,

$$
\left\langle\hat{a}_{R}(x, \omega)\right\rangle=e^{i k_{s p p} x}\left\langle\hat{a}_{R}(\omega)\right\rangle .
$$

The average of the megnetic field can be then derived,

$$
\begin{aligned}
& \left\langle\hat{H}^{+}(r, \omega)\right\rangle=-\left(\frac{\beta(\omega)}{2 k}\right)^{\frac{1}{2}} C^{\prime} e^{i k_{s p p} x}\left\langle\hat{a}_{R}(\omega)\right\rangle \times \\
& \left\{\left(\nu_{i}+\frac{k_{s p p}^{2}}{\nu_{i}}\right) e^{\left(\nu_{i} z\right)} \Theta(-z)-\left(\nu_{0}+\frac{k_{s p p}^{2}}{\nu_{0}}\right) e^{\left(-\nu_{0} z\right)} \Theta(z)\right\} .
\end{aligned}
$$

\section{B. Propagation in amplifying and attenuating media}

The ohmic loss property of the metal plays the main role in dissipation of SPP energy and reducing the its propagation length. To overcome these problems one uses a gain dielectric medium (with doped dye molecules) adjust to the metal medium. By this system not only the SPP loss can be compensated but also the SPP modes can be amplified[23-27]. 
On the other hand in (32), if the imaginary part is positive, then the SPP modes will be attenuated. In this condition the amplification in the dielectric can not compensate the loss of the metal. In contrast, if it is negative, the SPP modes can be amplified, because the gain of the dielectric can overcome the metal loss [28]. In order to investigate the magnetic field average (see Eq.(57)), we consider both attenuating and amplifying systems. Moreover, because of the quantum nature of (57) we can consider these conditions for two different kinds of SPP modes, i.e., coherent and squeezed states [29].

\section{1. coherent state of $S P P$}

In Ref. [29], it is mentioned that the SPP operators, like photon operators, satisfy the Bosonic commutation relations. Therefore, we can define the coherent states of SPP as eigenvector of its annihilation operator, in accordance with the definition of the coherent states of radiation field,

$$
\hat{a}_{R}(\omega)|\alpha\rangle=\alpha|\alpha\rangle
$$

By considering the attenuating system [28] and the equation (58), the variation of magnetic field average (see (57)) in coherent state is illustrated in Fig.(2).

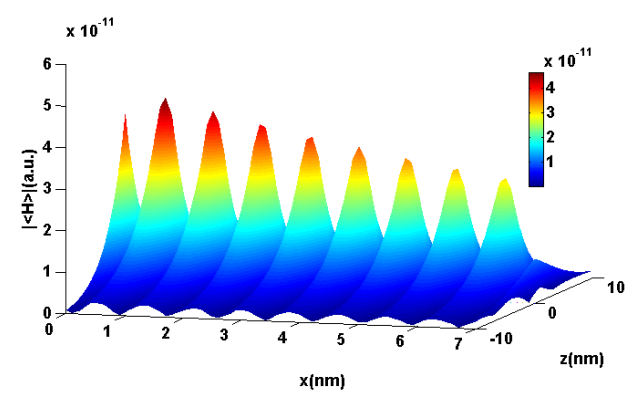

FIG. 2. The magnetic field propagation of SPP coherent state in an attenuating system. The dielectric medium with refractive index $n_{d}=1.3375-i 0.220$ and silver with $n_{m}=0.224+i 1.34$ at $\lambda=350 \mathrm{~nm}$ are chosen [28]. $k_{s p p}^{\prime \prime}=7.5 \times 10^{7}$ and $|\alpha|^{2}=7$.

Fig. (2) shows that for attenuating system, SPP propagation is attenuated because the dielectric gain can not compensate the dissipation in metal. On the other hand, for dielectric 
medium with refractive index $n_{d}=1.3375-i 0.223$ the imaginary part of the SPP refractive index is negative and $k_{s p p}^{\prime \prime}=-2.2376 \times 10^{7}$, so the system can be considered as an amplifying system. Fig.3 illustrates SPP magnetic field propagation in the amplifying system.

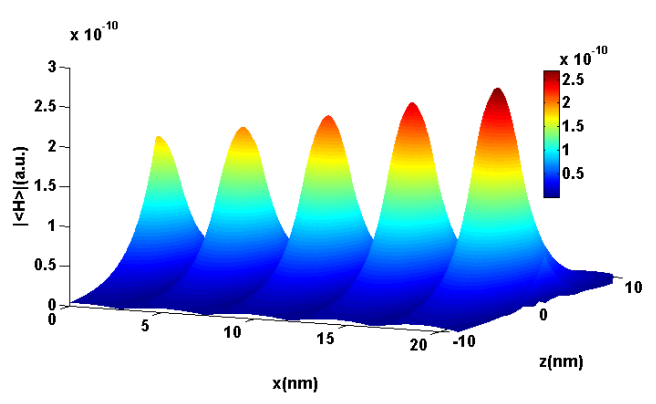

FIG. 3. The magnetic field propagation of SPP coherent state in an amplifying system.

Fig. (3) shows that SPP magnetic field along the propagated axis, can be amplified. It means that the gain of the dielectric has overcompensated the loss in the metal.

\section{Squeezed states of SPP}

After observing the quadrature squeezing of SPP in a gold waveguide [35], the quantum mechanical description of these states have been given [29]. The squeezed state of SPP is generated, similar to the squeezed state of radiation field, by applying the squeezed operator on vacuum,

$$
|\xi\rangle=\hat{s}(\xi)|0\rangle=\exp \left(\frac{1}{2} \xi^{*} \hat{a}^{2}-\frac{1}{2} \xi \hat{a}^{\dagger^{2}}\right)|0\rangle
$$

where $\xi=|\xi| e^{i \theta_{\xi}},|\xi|$ and $\theta_{\xi}$ are the squeezed parameter and compressed angle, respectively. If the SPP is prepared in the coherent squeezed state $\left|\xi, \alpha^{\prime}\right\rangle$, then

$$
\hat{a}(\omega)\left|\xi, \alpha^{\prime}\right\rangle=\left(\mu \alpha^{\prime}(\omega)-\nu \alpha^{\prime}(\omega)\right)\left|\alpha^{\prime}\right\rangle
$$

where $\mu=\cosh (|\xi|)$ and $\nu=\sinh (|\xi|) e^{i \theta_{\xi}}$. By applying (57) and (60), we can illustrate the magnetic field average of SPP squeezed state, as given for an attenuating system in Fig. (4).

This figure shows that, the propagation of the SPP will undergo more attenuation in any 


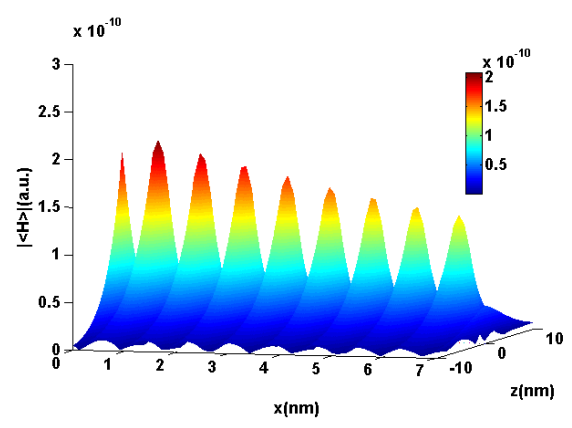

FIG. 4. The magnetic field propagation of SPP squeezed state in an attenuating system. $|\xi|=1.5$, $\theta_{\xi}=0, \alpha^{\prime}=|\alpha| e^{i \theta}$ and $\theta=1.5(\mathrm{rad})$.

state other than squeezed state, e.g., if the initial SPP state is prepared in squeezed state, the amplitude of the magnetic field is greater than the coherent state case (see Fig.2).

Likewise, the SPP magnetic field in an amplifying system and squeezed state is shown in Fig.5.

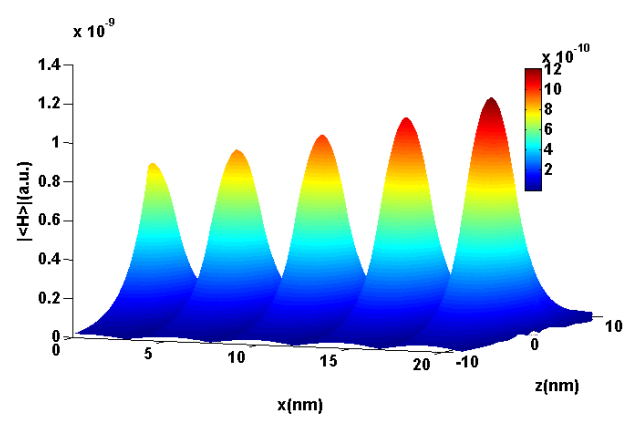

FIG. 5. The magnetic field propagation of SPP squeezed state in an amplifying system.

The comparison between Fig. (5) and Fig. (3) shows that those systems that the SPP modes is amplified, the magnitude of magnetic field in squeezed state is several times greater than in the coherent state case. 
3. comparison between SPP coherent and squeezed state in the amplifying system

In Ref. [36] the importance of the phase on the properties of a squeezed state, has been studied. Accordingly we can investigate the influence of the phase by considering the variation of the average of SPP magnetic field in (57) and (60) versus $\theta$, where $\theta$ is the argument of $\alpha^{\prime}=\left|\alpha^{\prime}\right| e^{i \theta}$. It is depicted in Fig. (6).

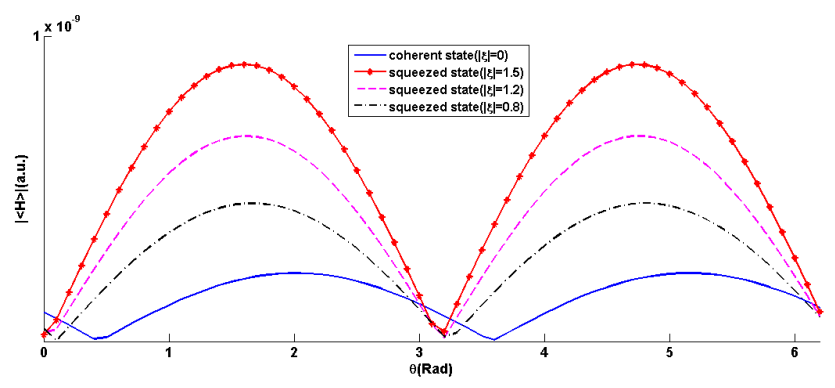

FIG. 6. The variation of SPP magnetic field average for different phases and different values of squeezed parameter, $z=0$ and $x=10 \mathrm{~nm}$.

As is illustrated in Fig. (6), the SPP magnetic field in squeezed state is greater than in the case of coherent state. The drastic difference between coherent and squeezed state is occurred in $\theta=1.5$ Rad. In Fig. (7) we demonstrate the SPP magnetic field for various parameters of squeezed states.

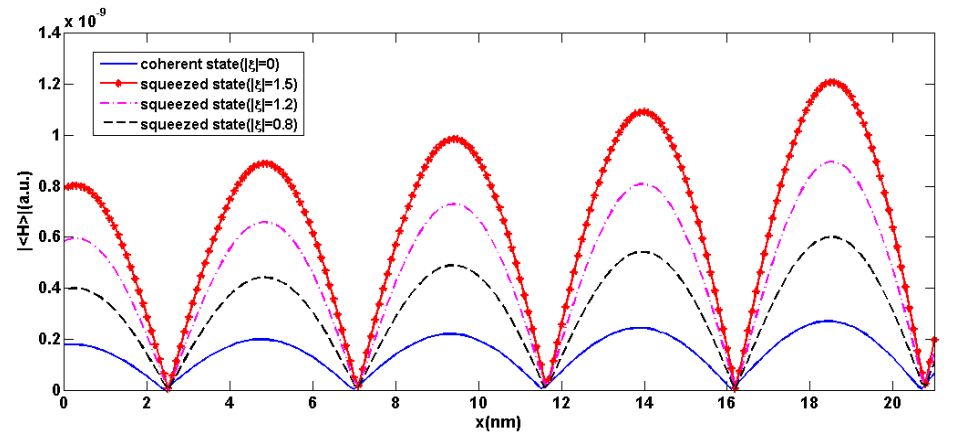

FIG. 7. The variation of SPP magnetic field average for different values of squeezed parameter, $z=0$ and $\theta=1.5 \operatorname{Rad}$. 
Fig. (7) shows that by choosing an appropriate phase, the magnetic of SPP in squeezed state is several times greater than that of coherent state.

\section{CONCLUSION}

The quantization of SPP by a new method, based on the Green's tensor, provides the possibility to distinguish the behavior of different modes of the quantized field, such as coherent and squeezed states of SPP. The propagation of different modes of quantized SPP can also be investigated in amplifying and attenuating media. We have seen that the behavior of these two modes in the said media are distinguished drastically.

We think, by extension of this method, the quantization of SPP in different geometries, other than the plane interfaces, can also be done straightforwardly, and accordingly it leads to understand the more interesting properties of SPP.

\section{APPENDIX A}

The Green's tensor satisfy the maxwell's equation when the source term is replaced by Dirac delta function( see Eq. (19)):

$$
-\nabla \times \nabla \times G\left(r, r^{\prime}, \omega\right)+\frac{\omega^{2}}{c^{2}} \epsilon(r, \omega) G\left(r, r^{\prime}, \omega\right)=I \delta\left(r-r^{\prime}\right) .
$$

For large frequency, $\epsilon(r, \omega) \rightarrow 1$ and from above equation one can deduce that:

$$
\lim _{|\omega| \rightarrow \infty} \frac{\omega^{2}}{c^{2}} G\left(r, r^{\prime}, \omega\right)=-\delta\left(r-r^{\prime}\right)
$$

The Green's tensor has also general properties such as:

$$
\begin{aligned}
& G^{T}\left(r, r^{\prime}, \omega\right)=G\left(r^{\prime}, r, \omega\right) \\
& G^{*}\left(r, r^{\prime}, \omega\right)=G\left(r, r^{\prime},-\omega\right)
\end{aligned}
$$

On the other hand for a nonmagnetic media,by some algebra, one can obtain a very usefull relation[37]

$$
\int \mathrm{d} s \operatorname{Im} \epsilon(s, \omega) G(r, s, \omega) \cdot G^{*}\left(s, r^{\prime}, \omega\right)=\frac{c^{2}}{\omega^{2}} \operatorname{Im} G\left(r, r^{\prime}, \omega\right)
$$




\section{APPENDIX B}

The right hand side of Eq.(41) can be express versus Green's tensor. By considering Eq.(23) and the Green's tensor for a metal-dielectric geometry Eq. (33), one can prove that:

$$
\begin{aligned}
& \int \mathrm{d} s \operatorname{Im} \epsilon(s, \omega) G(r, s, \omega) \cdot G^{*}\left(s, r^{\prime}, \omega\right)= \\
& \frac{\left|k_{s p p}\right|^{2}}{2 k^{\prime} k} \gamma(\omega)\left|C^{\prime}\right|^{2}\left\{\frac{e^{i k_{s p p}\left|x-x^{\prime}\right|}}{k_{s p p}}+\frac{e^{-i k_{s p p}^{*}\left|x-x^{\prime}\right|}}{k_{s p p}^{*}}\right\} \times \\
& {\left[\left(i \hat{x}+\frac{k_{s p p}}{\nu_{i}} \hat{z}\right) e^{\left(\nu_{i} z\right)} \Theta(-z)+\left(i \hat{x}-\frac{k_{s p p}}{\nu_{0}} \hat{z}\right) e^{\left(-\nu_{0} z\right)} \Theta(z)\right] \times} \\
& {\left[\left(-i \hat{x}+\frac{k_{s p p}^{*}}{\nu_{i}^{*}} \hat{z}\right) e^{\nu_{i}^{*} z^{\prime}} \Theta\left(-z^{\prime}\right)+\left(-i \hat{x}-\frac{k_{s p p}^{*}}{\nu_{0}^{*}} \hat{z}\right) e^{-\nu_{0}^{*} z^{\prime}} \Theta\left(z^{\prime}\right)\right],}
\end{aligned}
$$

where

$$
\gamma(\omega)=\left(1+\frac{\left|k_{s p p}\right|^{2}}{\left|\nu_{i}\right|^{2}}\right) \frac{\operatorname{Im} \epsilon_{m}}{\nu_{i}+\nu_{i}^{*}}+\left(1+\frac{\left|k_{s p p}\right|^{2}}{\left|\nu_{0}\right|^{2}}\right) \frac{\operatorname{Im} \epsilon_{d}}{\nu_{0}+\nu_{0}^{*}} .
$$

Equating Eq.(41) and Eq.(65) and considering Eq.(64) leads to:

$$
\left[\hat{A}(r, t),-\epsilon_{0} \hat{E}\left(r^{\prime}, t\right)\right]=\int \mathrm{d} \omega \frac{i \omega \epsilon_{0} \mu_{0}^{2} \beta(\omega)}{\pi \gamma(\omega)} \frac{c^{2}}{\omega^{2}} \operatorname{Im} G\left(r, r^{\prime}, \omega\right) .
$$

[1] D. k. Gramotnev and S. I. Bozhevolnyi," Plasmonic beyond the differaction limit," Nature Photon. 4, 83 (2010).

[2] J. Takahara, ..." "Opt. Lett. 22, 475 (1997).

[3] J. A. Schuller, E. S. Banard, W. Cai, Y. C. Jun, J. S. White and M. I. Brongersma," Plasmonic for extreme light concentration and manipulation." Nat. Mater., 9193 (2010).

[4] E. Altewischer, M. P. Van Exter, J. P. Woerdman, " Plasmon assisted transmission of entangled photons." Nature, 418, 304 (2002).

[5] E. Moreno, F. J. Garcia Vidal, D Erni, ..." Theory of plasmon assisted transmission of entangled photons." Phys. Rev. Lett, 92 236801(2004).

[6] A. Huck, S. Smolka, P. Lodahl, ..."Demonstration of quadrature squeezed surface plasmons in a gold waveguide." Phys. Rev. Lett, 102 246802(2009).

[7] Y. LiHua, W. YongGang and Y. Bojun," Description of squeezed surface plasmons." Sci ChinaPhys Mech Astorn, 541583(2011) 
[8] J. L. Van Velsen, J. Tworzyldo and C. W. J. Beenakker, "Scattering theory of plasmon-assisted entanglement transfer and distillation." Phys. Rev. A, 68043807 (2003).

[9] D. E. Chang, A. S. Sørensen, P. R. Hemmer and M. D. Lukin," Quantum Optics with Surface Plasmons." Phys. Rev. Lett., 97053002 (2006).

[10] D. E. Chang, A. S. Sørensen, E. A. Demler and M. D. Lukin," A single photon transistor using nanoscale surface plasmons." Nature Phys., 3 807(2007).

[11] D. Ballester, M. S. Tame, C. Lee, J. Lee and M. S. Kim," Long-range surface plasmon polariton excitation at the quantum level." Phys. Rev. A79 053845 (2009).

[12] D. Ballester, M. S. Tame and M. S. Kim,"Quantum theory of surface-plasmon polariton scattering." Phys. Rev. A82 012325 (2010).

[13] J. M. Elson and Ritchie,"Photon Interactions at a Rough Metal Surface." Phys. Rev. B, 4 4129(1971).

[14] B. Huttner and S. M. Barnett,"Q uantization of the electromagnetic field in dielectric." Phys. Rev. A 464306 (1992).

[15] R. Matloob, R. Loudon, S. M. Barnett and J. Jeffers,"Electromagnetic field quantization in absorbing dielectrics" Phys. Rev. A 52 4823,(1995).

[16] T. Gruner and D.-G. Welsch," Green-function approach to the radiation-field quantization for homogeneous and inhomogeneous Kramers-Kronig dielectrics." Phys. Rev. A 531818 (1996)

[17] L. Knöll, S. Scheel, D.-G. Welsch, QED in dispersing and absorbing media:coherent and Statistics of Photons and Atoms edited by J. Perina (John Wiley \& Son, New York, 2001).

[18] A. Tip, L. Knöll, S. Scheel, and D.-G. Welsch,"On the equivalence of the Langevin and auxiliary field quantization methods for absorbing dielectrics." 63043806 (2001).

[19] H. T. Dung, L. Knöll and D.-G. Welsch,"Three-dimensional quantization of the electromagnetic field in dispersive and absorbing inhomogeneous dielectrics." Phys. Rev. A 573931 (1998).

[20] M. Amooshahi and F. Kheirandish,"Electromagnetic field quantization in an anisotropic magnetodielectric medium with spatial-temporal dispersion.” J. Phys. A: Math. Gen. 41275402 (2008).

[21] M. Amooshahi and B. N. Esfahani," Canonical quantization of the electromagnetic field in an anisotropic polarizable and magnetizable medium." Ann. Phys. 3251913 (2010). 
[22] C. Raabe and D.-G. Welsch,"QED in arbitrary linear media: amplifying media." Eur. Phys. J. Spec. Top. 160371 (2008).

[23] T. Zhang, and F. Shan." Development and application of surface plasmon polaritons on optical amplification." Journal of Nanomaterials 2014 (2014).

[24] I. Suarez, E. P. Fitrakis, P. Rodriguez-Canto, R. Abargues, I. Tomkos and J. Martinez-Pastor, (2012, July). "Surface plasmon-polariton amplifiers." In Transparent Optical Networks (ICTON), 2012 14th International Conference on (pp. 1-5). IEEE.

[25] M. A. Noginov, V. A. Podolskiy, G. Zhu, M. Mayy, M. Bahoura, J. A. Adegoke, B. A. Ritzo, and K. Reynolds. "Compensation of loss in propagating surface plasmon polariton by gain in adjacent dielectric medium." Optics express 161385 (2008).

[26] M. A. Noginov, G. Zhu, M. Bahoura, J. Adegoke, C. E. Small, B. A. Ritzo, V. P. Drachev, and V. M. Shalaev. "Enhancement of surface plasmons in an Ag aggregate by optical gain in a dielectric medium." Optics letters. 313022 (2006).

[27] P. Berini, and I. De Leon. "Surface plasmon-polariton amplifiers and lasers." Nature Photonics. 616 (2012).

[28] I. Avrutsky. "Surface plasmons at nanoscale relief gratings between a metal and a dielectric medium with optical gain.” Phys. Rev. B 70, 155416 (2004).

[29] L. Yang, Y. Wang and B. Yang," Description of squeezed surface plasmons." Science China Physics, Mechanics and Astronomy, 541583 (2011).

[30] H. M. Nussenzveig, Causality and dispersion relations(New York: Academic, 1972).

[31] L. Landau, E. M. Lifshitz and L. P. Piaevskii, Electrodynamics of Continuous Media 2nd ed. (Oxford, 1984).

[32] T. Söndergaard and B. Tromborg," General theory for spontaneous emission in active dielectric microstructures: Example of a fiber amplifier." Phys. Rev. A 64033812 (2001).

[33] T. Söndergaard and S. I. Bozhevolnyi," Surface plasmon polariton scattering by a small particle placed near a metal surface: An analytical study." Phys. Rev. B 69045422 (2004).

[34] J. Jung and T. Söndergaard," Greens function surface integral equation method for theoretical analysis of scatterers close to a metal interface." Phys. Rev. B 77245310 (2008).

[35] A. Huck, S. Smolka, P. Lodahl, A. S. Sørensen, A. Boltasseva, J. Janousek, and U. L. Andersen, " Demonstration of quadrature-squeezed surface plasmons in a gold waveguide." Phys. Rev. Lett. 102, 246802 (2009). 
[36] W. Vogel and D. G. Welsch. Quantum optics(John Wiley \& Sons, 2006).

[37] S. Y. Buhmann, Dispersion Force I(Springer, Berlin, 2012). 